Sharif University of Technology
Scientia Iranica
SCIENTIA
I RAN ICA
http://scientiairanica.sharif.edu

Research Note

\title{
Field study of flow resistance in step-pool streams (case study of Dizin River)
}

\author{
A. Tahershamsi ${ }^{a, *}$, M.R. Majdzadeh Tabatabai ${ }^{b}$, and A. Torabizadeh ${ }^{a}$ \\ a. Faculty of Civil and Environmental Engineering, Amirkabir University of Technology, Tehran, Iran. \\ b. Faculty of Water and Environmental Engineering, Shahid-Beheshti University, Tehran, Iran.
}

Received 6 April 2016; received in revised form 8 January 2017; accepted 30 April 2018

\section{KEYWORDS}

Step-pool;

Flow resistance;

Mountain streams;

Chezy coefficient;

Dizin River.

\begin{abstract}
A series of steps and pools are ubiquitous bed forms in mountain stream channels, occurring where gradients exceed $2 \%$ and materials are in the gravel to boulder size range. Flow resistance, reflected by roughness elements, appears to be an important controlling factor in bed load transport rates and mean flow velocity. To estimate flow resistance, some morphological features and velocity were measured in the step-pool channel of Dizin River, located in Karaj River watershed in Iran. Topographic surveys and bed sediment sampling were made in a low-flow condition, while three-dimensional velocity measurements were made in low, medium, and high flow conditions. Gradient variations are in the range of $7 \%$ to $14 \%$. As flow resistance is a function of geometry, bed material size, longitudinal slope, and hydraulic radius, dimensional analysis was conducted to develop a non-dimensional relationship for flow resistance in step-pool reaches. Thereafter, it was calibrated for the measured dataset of Dizin river and validated for Rio Cordon dataset. Comparable results of validation with a river located in a different environment suggest that flow resistance features in semi-arid and humid streams may have similar effects on a non-dimensional resistance coefficient.
\end{abstract}

(C) 2018 Sharif University of Technology. All rights reserved.

\section{Introduction}

Step-pool and cascade bed forms were identified at low discharges following the morphological classification of Montgomery and Buffington (1997). In their classification, step-pool channels are characterized by longitudinal steps formed by large clasts organized into discrete channel-spanning accumulations that separate pools containing finer materials. Typically, cascades are longitudinally and laterally disorganized bed materials, generally consisting of individual cobbles and boulders;

\footnotetext{
*. Corresponding author.

E-mail address: tshamsi@aut.ac.ir (A. Tahershamsi)
}

they are separated by small, partially channel-spanning pools [1]. In a classification made by Grant et al. (1990), step-pools and cascades operate differently, whereas the step-pools of Chin (1999) include both cascades and step-pools. The stepped morphology of the bed results in alternating between critical to supercritical flow over steps and subcritical flow in pools. Step-pools represent a typical bed morphology in streams exceeding $\sim 2-3 \%$ gradient [1-3]. Steppool morphology is associated generally with steep gradients, small width-to-depth ratios, and pronounced confinement by valley walls. Although step-forming clast sizes are typically comparable to annual high flow depths, a stepped longitudinal profile also may develop in steep sand-bedded channels [4]. Chin (1989) suggested that step-pools can be recognized by their 
staircase-like longitudinal profile resulting from the accumulation of cobbles and boulders, which are located transversely across the channel, alternating with pools containing finer sediments. He also stated that step-pools changed the path of water and sediments from up-land to low-land basins [5]. This, in turns, received a great deal of support by many authors to maintain up-land aquatic ecosystems [6-8]. Chin et al. (2009) concluded that reproducing the physical properties of step-pool reach may ensure maximum stability for a restored stream profile [9]. Artificial steppools can be used to introduce soft restoration methods where concrete has long been used. Therefore, in this context, Nikseresht et al. (2013) used the structures of step-pools to study two-phase flow in spillways numerically [10].

Morphological and hydraulic approaches have been adopted to model step-pool properties [11]. The hydraulic approach consists of investigating the equations governing flow and sediment transport through step-pools $[12,13]$. However, step-pools challenge researchers in many of their presented aspects. This may be due to a consistent interaction between steppool hydraulics and bed form variations, which may make the validity of available flow resistance equations for gravel and cobble bed rivers debatable [14-16]. These problems explain the reasons why morphological approaches have been widely used in stream restoration in association with hydraulics [8]. In this approach, various relations link the step-pool dimensions (length and height) to other geometric parameters [17]. Most geometric equations have focused on slope and boulders' diameter; however, other parameters, such as the active width [18] and woody debris density [19-22], have been found to be playing an important role in the steppool morphology.

To understand step-pool formation processes and hydraulic controls, many researchers have considered step-pool geometry through relationships between step length $(L)$, step height $\left(H_{s}\right)$, grain size diameter $(D)$, stream gradient $(S)$, and channel width $(w)$ [6,23]. Step-pool channels are characterized by their range of slopes between 0.03 and $0.07 \mathrm{~m} / \mathrm{m}$ [1] and step elevations to control energy dissipation [24].

The ratio of step height to step length, $H_{s} / L / S$, followed by bed gradient is an often-cited measure of step geometry that illustrates the amount of the elevation change created by step-pool sequences and the presence of reverse slopes between steps [25]. Abrahams et al. (1995) suggested that step-pool channels were ideally organized such that flow resistance would be maximized, and this could be achieved only if $1<$ $((H / L) / S)<2[26]$.

Studies of flow resistance dynamics have explored methods to predict roughness coefficients as a function of factors such as relative submergence [27], step ge- ometry [14], or unit discharge and hydraulic geometry $[13,16,28,29]$. Based on flume experiments, Wilcox et al. (2006) concluded that the combined effects of woody debris and spill resistance dominated total flow resistance in step-pool channels [22], whereas grain resistance was relatively low. Observations of elevated sediment transport rates following an exceptional flood that destroyed steps in the Erlenbach, Switzerland, illustrated how formed resistance could decrease as a result of step destruction [30]. Zimmermann (2010), however, suggested that stress partitioning was inappropriate for steep channels, where grains actually induce formed resistance, as opposed to the skin resistance associated with grains in lower-gradient systems [16].

D'Agostino and Michelini (2015) used his own database to verify velocity estimation relationships developed by other researchers in the step-pool reach of mountain streams. Flow regime and structure have been studied by a number of researchers [31]. Sindelar and Smart (2016) argued that flow regime was related to the mean value of the Froude Number in a step-pool reach, while Maddahi et al. (2016) studied the effect of bed form variations on the flow structure $[32,33]$.

In this paper, attempts are made to gain an indepth understanding of mountain streams in a semiarid environment. This is achieved by field surveys in Dizin River, located in Iran. The collected data are added to the database gathered from other articles to obtain a more generalized relationship for flow resistance estimation.

\section{Methods}

\subsection{Study area}

Our study area is Dizin River in Iran. It is one of the main branches of Karaj River, originating from the south flank of Alborz Mountain in $60 \mathrm{~km}$ west of Tehran from Kharsang Kuh Watershed (Kolun Bastak) (Figures 1 and 2). Snowmelt generates late spring/early summer peak flows. Water discharge and bed material sampling are measured in Dizin River. The study site is evaluated to be approximately 3900 $\mathrm{m}$ above the sea level, with a drainage area of $10 \mathrm{~km}^{2}$.

The study reach is sinuous with the length 12 times the average bankfull width of 1.27 to 8.2 along which 12 steps are observed clearly; it is divided into 4 subreaches with 3 steps in each reach. Gradient variations are identified to be in the range of $7 \%$ to $14 \%$. Bed sediments are typically poorly imbricated and weakly rounded, and steps are formed by irregular accumulation of boulders across the channel. Median grain size in the 4 subreaches ranges from 140 to $220 \mathrm{~mm}$ with $D_{84}$ from 410 to $480 \mathrm{~mm}$. No woody debris is observed in the study reach. 


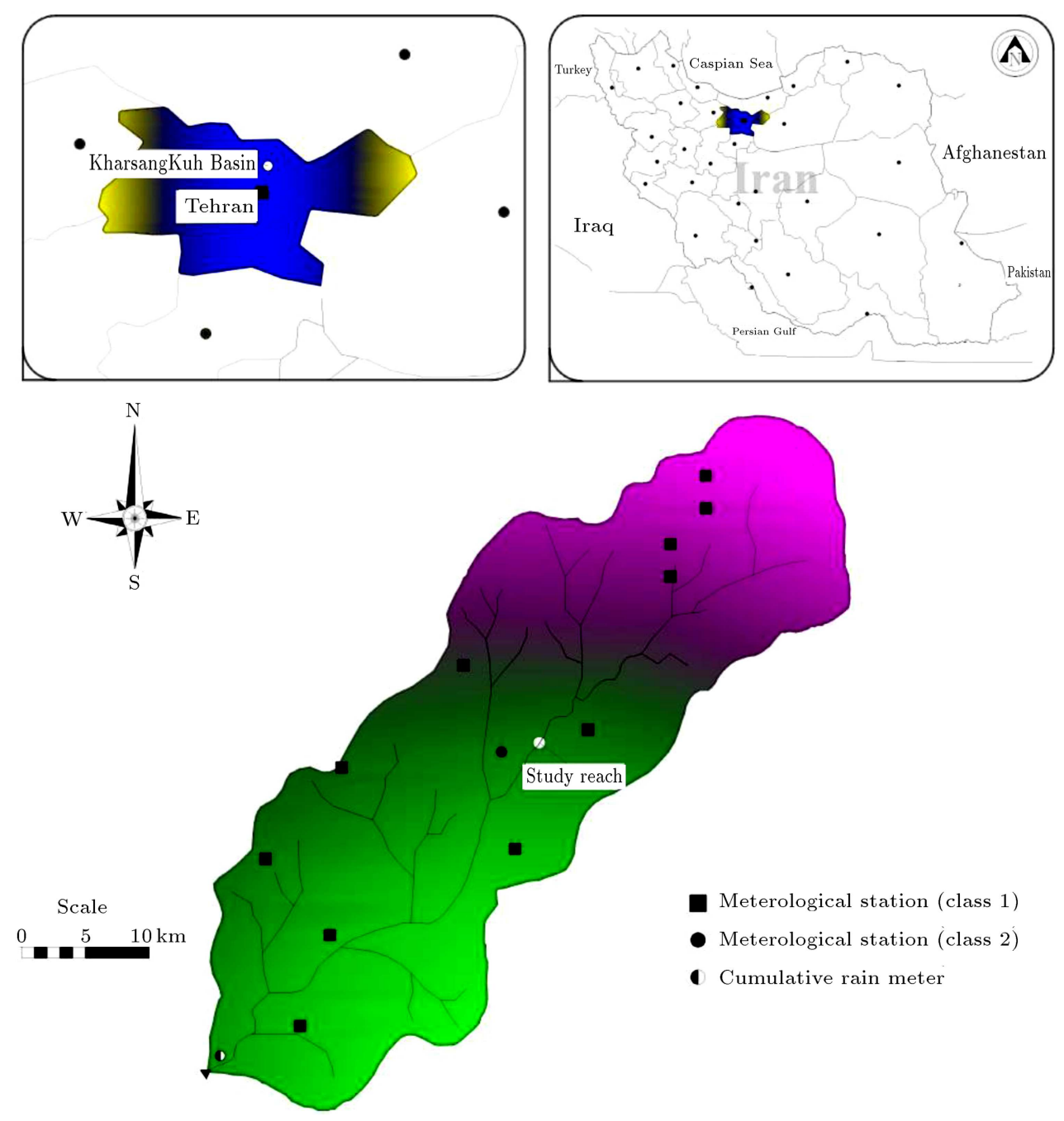

Figure 1. Study area of Dizin step-pool.

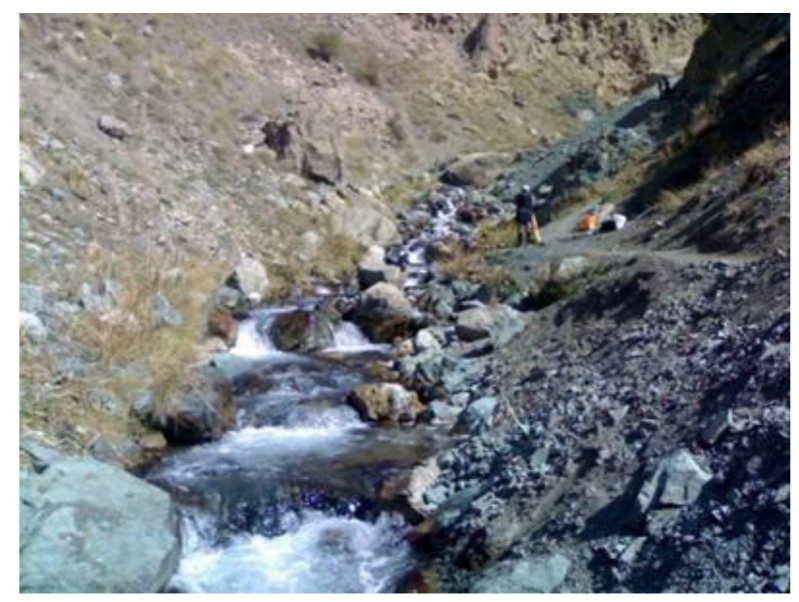

Figure 2. An overall view of Dizin Reach and mapping stations.

\subsection{Field measurements}

River survey was made along $120 \mathrm{~m}$ of the study reach with the width of $10 \mathrm{~m}$. Total station was used to survey the area with 2310 topographic points (Figure 3 ).

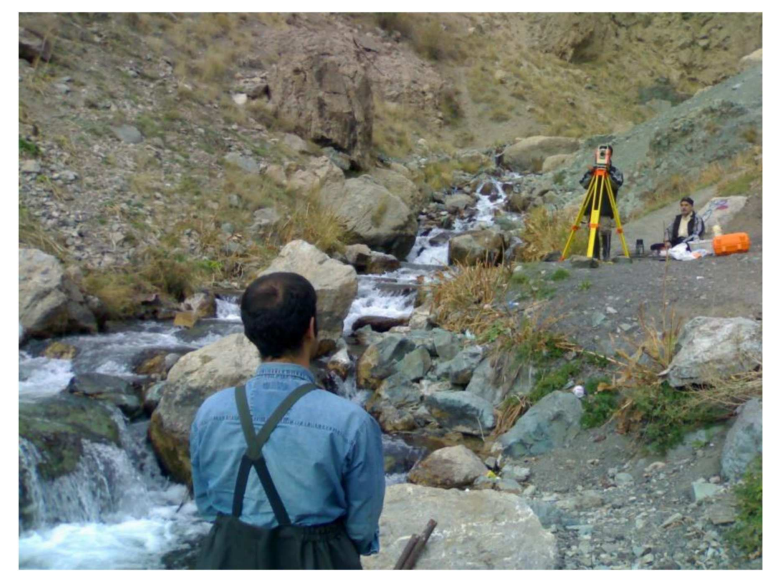

Figure 3. Topography measurements.

Topographic points were selected by considering several characteristics such as crest and bottom of the steps, breaks in the slope, reach's uniformity, and isolated boulders in bed. Distance between points varied from $30 \mathrm{~cm}$ to $2 \mathrm{~m}$. Analogous maps were established to 
extract 33 cross-sections from the topographic survey (Figure 4). Since there was no significant changes in river bed during field surveys, one representative longitudinal profile of the river bed was drawn.

Data collections were made in three different dates to monitor river discharges in different conditions of low, medium, and high flows. In the first field survey, bed topographic and water surface profile measurements were made simultaneously, while water surface profiles were the only measurements in other occasions (Tables 1-3).

Velocity measurements were made across two cross-sections: upstream and downstream of the reach (Figure 5). These measurements were made in the first field survey by current meters and by Acoustic Doppler Velocimeter (ADV) in other occasions [34,35]. This instrument is always used to record flow velocity at a minimum distance of $50 \mathrm{~mm}$ above the bed and below water surfaces. The continuity equation was then applied to estimate water discharge on the day of the survey.

Cross-section geometry and flow characteristics are derived from Figure 4 for each discharge measurement (Tables 1-3).

Bed material samplings were made on the date when flow was in its lowest condition. They were made as surface sampling based on Wolman (1954) at two different cross-sections which were $20 \mathrm{~m}$ apart [36]. Subsurface sampling was also made at the above sections by using a CSU barrel to avoid water perturbation during the sampling procedure [37] (Table 4).

In rivers with coarse materials, $R / D_{84}$ should be considered in flow resistance equations [38]. This ratio is applied with $D_{84}$; as in coarse bed rivers, due to

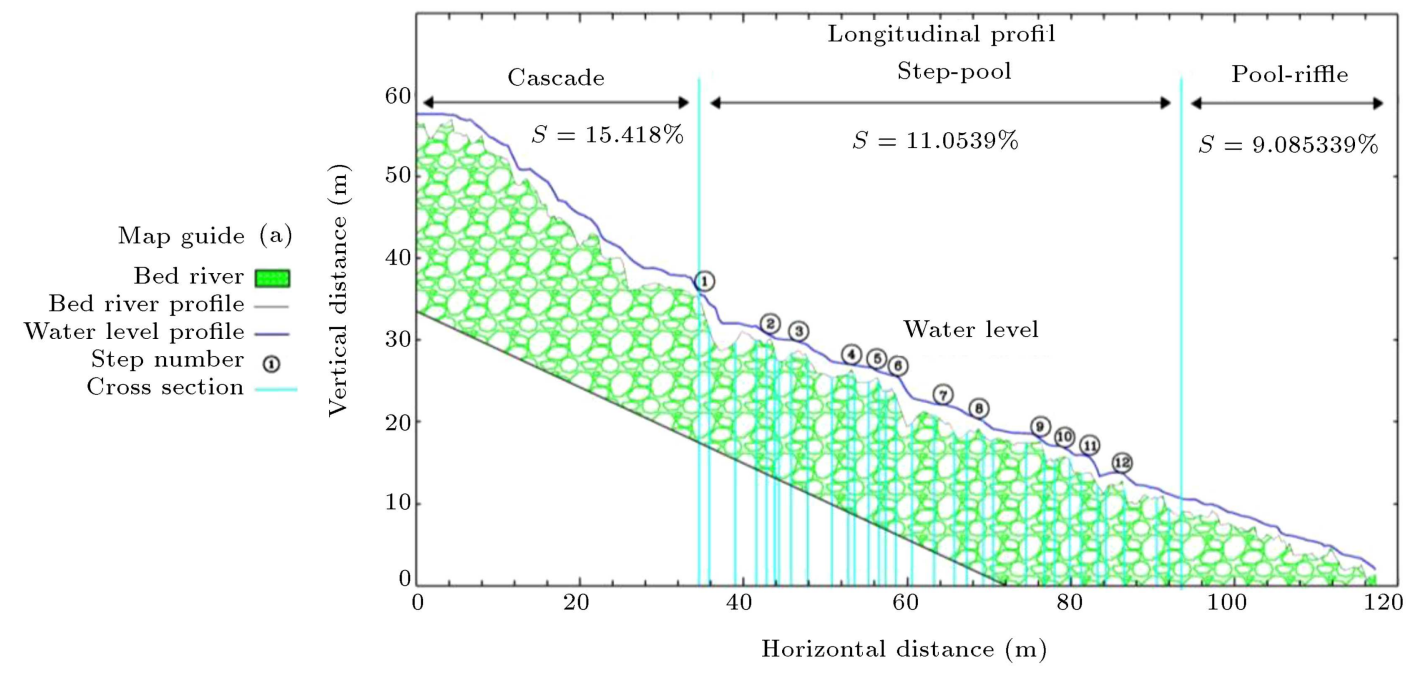

(a)
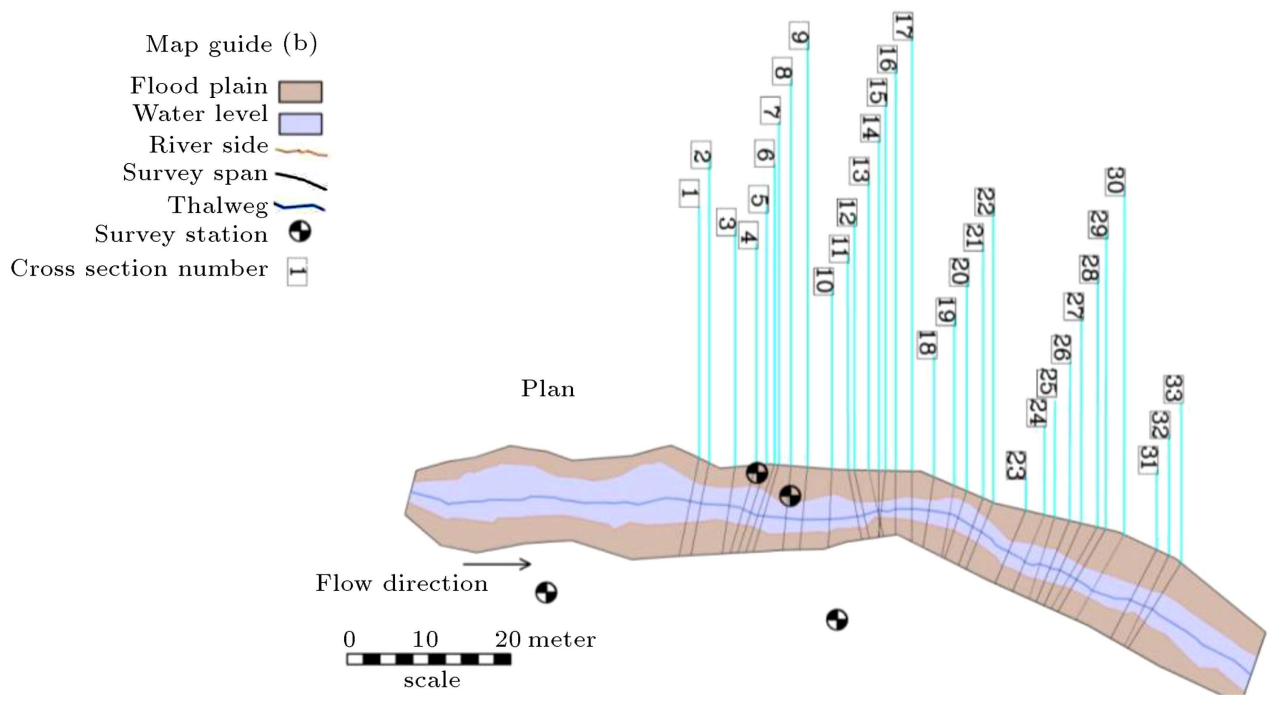

(b)

Figure 4. Cross section of Dizin step-pool. 
Table 1. Cross-section geometry and flow characteristics for $0.399 \mathrm{~m}^{3} / \mathrm{s}$ discharge (dated 3-Nov-2009).

\begin{tabular}{|c|c|c|c|c|c|c|c|c|}
\hline Subreach & $\begin{array}{c}\text { Section } \\
\text { no. }\end{array}$ & $\begin{array}{l}\text { Area } \\
\left(\mathrm{m}^{2}\right)\end{array}$ & $\begin{array}{c}\text { Wetted } \\
\text { perimeter } \\
(\mathbf{m})\end{array}$ & $\begin{array}{c}\text { Top } \\
\text { width } \\
(\mathrm{m}) \\
\end{array}$ & $\begin{array}{c}\text { Hydraulic } \\
\text { radius } \\
(\mathrm{m})\end{array}$ & $\begin{array}{c}\text { Hydraulic } \\
\text { depth } \\
(\mathrm{m})\end{array}$ & $\begin{array}{c}\text { Mean } \\
\text { velocity } \\
(\mathrm{m} / \mathrm{s})\end{array}$ & $\begin{array}{c}\text { Froude } \\
\text { no. }\end{array}$ \\
\hline \multirow{10}{*}{1} & 1 & 0.306 & 3.385 & 1.601 & 0.172 & 0.191 & 1.304 & 0.952 \\
\hline & 2 & 0.501 & 3.115 & 1.280 & 0.273 & 0.392 & 0.796 & 0.406 \\
\hline & 3 & 1.502 & 9.787 & 4.772 & 0.300 & 0.315 & 0.266 & 0.151 \\
\hline & 4 & 1.091 & 10.956 & 5.419 & 0.197 & 0.201 & 0.366 & 0.260 \\
\hline & 5 & 1.403 & 9.382 & 4.579 & 0.292 & 0.307 & 0.284 & 0.164 \\
\hline & 6 & 1.477 & 10.267 & 4.808 & 0.271 & 0.307 & 0.270 & 0.156 \\
\hline & 7 & 1.732 & 10.662 & 5.133 & 0.313 & 0.337 & 0.230 & 0.127 \\
\hline & 8 & 2.162 & 14.576 & 7.700 & 0.314 & 0.281 & 0.185 & 0.111 \\
\hline & 9 & 1.601 & 13.677 & 6.631 & 0.227 & 0.241 & 0.249 & 0.162 \\
\hline & 10 & 1.083 & 10.717 & 5.138 & 0.194 & 0.211 & 0.369 & 0.256 \\
\hline \multirow{8}{*}{2} & 11 & 1.080 & 7.342 & 3.600 & 0.288 & 0.300 & 0.370 & 0.216 \\
\hline & 12 & 0.306 & 4.038 & 1.826 & 0.138 & 0.167 & 1.306 & 1.020 \\
\hline & 13 & 1.162 & 9.097 & 4.278 & 0.241 & 0.272 & 0.344 & 0.211 \\
\hline & 14 & 0.812 & 8.772 & 4.151 & 0.176 & 0.196 & 0.491 & 0.355 \\
\hline & 15 & 1.259 & 10.358 & 4.777 & 0.226 & 0.264 & 0.317 & 0.197 \\
\hline & 16 & 1.057 & 9.710 & 4.515 & 0.204 & 0.234 & 0.377 & 0.249 \\
\hline & 17 & 2.382 & 9.870 & 4.610 & 0.453 & 0.517 & 0.168 & 0.074 \\
\hline & 18 & 0.566 & 4.925 & 2.376 & 0.222 & 0.238 & 0.705 & 0.461 \\
\hline \multirow{5}{*}{3} & 19 & 1.194 & 6.626 & 3.149 & 0.343 & 0.379 & 0.334 & 0.173 \\
\hline & 20 & 1.060 & 6.167 & 2.924 & 0.327 & 0.363 & 0.376 & 0.200 \\
\hline & 21 & 0.596 & 4.224 & 1.933 & 0.260 & 0.308 & 0.670 & 0.386 \\
\hline & 22 & 0.285 & 3.301 & 1.576 & 0.165 & 0.181 & 1.400 & 1.051 \\
\hline & 23 & 0.712 & 8.237 & 4.075 & 0.171 & 0.175 & 0.561 & 0.429 \\
\hline \multirow{10}{*}{4} & 24 & 1.124 & 10.252 & 4.983 & 0.213 & 0.226 & 0.355 & 0.239 \\
\hline & 25 & 0.802 & 9.097 & 4.495 & 0.174 & 0.178 & 0.498 & 0.376 \\
\hline & 26 & 0.757 & 5.415 & 2.548 & 0.264 & 0.297 & 0.527 & 0.309 \\
\hline & 27 & 0.423 & 6.657 & 3.243 & 0.124 & 0.130 & 0.945 & 0.836 \\
\hline & 28 & 0.721 & 7.157 & 3.471 & 0.195 & 0.208 & 0.554 & 0.388 \\
\hline & 29 & 0.816 & 7.266 & 3.528 & 0.218 & 0.231 & 0.489 & 0.325 \\
\hline & 30 & 0.823 & 7.619 & 3.477 & 0.199 & 0.237 & 0.485 & 0.318 \\
\hline & 31 & 0.455 & 4.486 & 2.106 & 0.191 & 0.216 & 0.878 & 0.603 \\
\hline & 32 & 0.516 & 4.219 & 2.003 & 0.233 & 0.257 & 0.774 & 0.487 \\
\hline & 33 & 0.704 & 6.864 & 3.202 & 0.192 & 0.220 & 0.567 & 0.386 \\
\hline
\end{tabular}


Table 2. Cross-section geometry and flow characteristics for $0.629 \mathrm{~m}^{3} / \mathrm{s}$ discharge (dated 14-Oct-2008).

\begin{tabular}{|c|c|c|c|c|c|c|c|c|}
\hline Subreach & $\begin{array}{c}\text { Section } \\
\text { no. }\end{array}$ & $\begin{array}{l}\text { Area } \\
\left(\mathrm{m}^{2}\right)\end{array}$ & $\begin{array}{c}\text { Wetted } \\
\text { perimeter } \\
(\mathrm{m})\end{array}$ & $\begin{array}{c}\text { Top } \\
\text { width } \\
(\mathbf{m})\end{array}$ & $\begin{array}{c}\text { Hydraulic } \\
\text { radius } \\
(\mathbf{m})\end{array}$ & $\begin{array}{c}\text { Hydraulic } \\
\text { depth } \\
(\mathbf{m})\end{array}$ & $\begin{array}{c}\text { Mean } \\
\text { velocity } \\
(\mathrm{m} / \mathrm{s})\end{array}$ & $\begin{array}{c}\text { Froude } \\
\text { no. }\end{array}$ \\
\hline \multirow{10}{*}{1} & 1 & 0.389 & 3.754 & 1.773 & 0.196 & 0.219 & 1.618 & 1.103 \\
\hline & 2 & 0.544 & 3.251 & 1.334 & 0.284 & 0.408 & 1.156 & 0.578 \\
\hline & 3 & 1.642 & 10.080 & 4.906 & 0.317 & 0.335 & 0.383 & 0.211 \\
\hline & 4 & 1.353 & 11.916 & 5.889 & 0.225 & 0.230 & 0.464 & 0.309 \\
\hline & 5 & 1.727 & 9.907 & 4.822 & 0.340 & 0.358 & 0.364 & 0.194 \\
\hline & 6 & 1.837 & 10.679 & 4.986 & 0.323 & 0.368 & 0.342 & 0.180 \\
\hline & 7 & 2.115 & 11.037 & 5.289 & 0.368 & 0.400 & 0.297 & 0.150 \\
\hline & 8 & 2.696 & 15.839 & 7.700 & 0.331 & 0.350 & 0.233 & 0.126 \\
\hline & 9 & 2.101 & 14.170 & 6.854 & 0.287 & 0.307 & 0.299 & 0.172 \\
\hline & 10 & 1.492 & 11.625 & 5.571 & 0.247 & 0.268 & 0.421 & 0.260 \\
\hline \multirow{8}{*}{2} & 11 & 1.080 & 8.311 & 3.770 & 0.238 & 0.286 & 0.582 & 0.347 \\
\hline & 12 & 0.560 & 4.836 & 2.181 & 0.211 & 0.257 & 1.123 & 0.708 \\
\hline & 13 & 1.507 & 9.990 & 4.664 & 0.283 & 0.323 & 0.417 & 0.234 \\
\hline & 14 & 1.327 & 10.126 & 4.761 & 0.247 & 0.279 & 0.474 & 0.286 \\
\hline & 15 & 1.646 & 11.383 & 5.227 & 0.267 & 0.315 & 0.382 & 0.217 \\
\hline & 16 & 1.385 & 10.463 & 4.875 & 0.248 & 0.284 & 0.454 & 0.272 \\
\hline & 17 & 2.908 & 11.382 & 5.329 & 0.480 & 0.546 & 0.216 & 0.093 \\
\hline & 18 & 0.754 & 5.834 & 2.814 & 0.250 & 0.268 & 0.834 & 0.514 \\
\hline \multirow{5}{*}{3} & 19 & 1.417 & 7.072 & 3.353 & 0.381 & 0.423 & 0.444 & 0.218 \\
\hline & 20 & 1.321 & 6.666 & 3.144 & 0.375 & 0.420 & 0.476 & 0.234 \\
\hline & 21 & 0.743 & 4.751 & 2.173 & 0.288 & 0.342 & 0.846 & 0.462 \\
\hline & 22 & 0.429 & 4.037 & 1.915 & 0.202 & 0.224 & 1.466 & 0.989 \\
\hline & 23 & 1.055 & 1.055 & 4.356 & -0.320 & 0.242 & 0.596 & 0.387 \\
\hline \multirow{10}{*}{4} & 24 & 1.474 & 10.761 & 5.203 & 0.265 & 0.283 & 0.426 & 0.256 \\
\hline & 25 & 1.085 & 9.697 & 4.781 & 0.221 & 0.227 & 0.579 & 0.388 \\
\hline & 26 & 0.738 & 7.505 & 3.608 & 0.189 & 0.204 & 0.852 & 0.602 \\
\hline & 27 & 0.885 & 9.820 & 4.815 & 0.177 & 0.184 & 0.710 & 0.529 \\
\hline & 28 & 0.896 & 8.005 & 3.888 & 0.218 & 0.231 & 0.701 & 0.466 \\
\hline & 29 & 1.023 & 7.656 & 3.704 & 0.259 & 0.276 & 0.614 & 0.373 \\
\hline & 30 & 1.048 & 8.020 & 3.652 & 0.240 & 0.287 & 0.600 & 0.357 \\
\hline & 31 & 0.705 & 8.238 & 3.975 & 0.165 & 0.177 & 0.891 & 0.676 \\
\hline & 32 & 0.987 & 6.977 & 3.332 & 0.271 & 0.296 & 0.637 & 0.374 \\
\hline & 33 & 1.026 & 7.681 & 3.575 & 0.250 & 0.287 & 0.613 & 0.365 \\
\hline
\end{tabular}


Table 3. Cross-section geometry and flow characteristics for $0.822 \mathrm{~m}^{3} / \mathrm{s}$ discharge (dated 28-Jul-2009).

\begin{tabular}{|c|c|c|c|c|c|c|c|c|}
\hline Subreach & $\begin{array}{c}\text { Section } \\
\text { no. }\end{array}$ & $\begin{array}{l}\text { Area } \\
\left(\mathrm{m}^{2}\right)\end{array}$ & $\begin{array}{c}\text { Wetted } \\
\text { perimeter } \\
(\mathrm{m})\end{array}$ & $\begin{array}{c}\text { Top } \\
\text { width } \\
(\mathrm{m})\end{array}$ & $\begin{array}{l}\text { Hydraulic } \\
\text { radius } \\
(\mathrm{m})\end{array}$ & $\begin{array}{c}\text { Hydraulic } \\
\text { depth } \\
(\mathrm{m})\end{array}$ & $\begin{array}{c}\text { Mean } \\
\text { velocity } \\
(\mathrm{m} / \mathrm{s})\end{array}$ & $\begin{array}{c}\text { Froude } \\
\text { no. }\end{array}$ \\
\hline \multirow{10}{*}{1} & 1 & 0.581 & 4.449 & 2.088 & 0.246 & 0.278 & 1.416 & 0.857 \\
\hline & 2 & 0.677 & 3.597 & 1.470 & 0.318 & 0.461 & 1.214 & 0.571 \\
\hline & 3 & 2.166 & 10.840 & 5.252 & 0.388 & 0.412 & 0.379 & 0.189 \\
\hline & 4 & 1.928 & 12.944 & 6.382 & 0.294 & 0.302 & 0.426 & 0.248 \\
\hline & 5 & 2.233 & 10.780 & 5.209 & 0.401 & 0.429 & 0.368 & 0.180 \\
\hline & 6 & 2.362 & 11.245 & 5.228 & 0.393 & 0.452 & 0.348 & 0.165 \\
\hline & 7 & 2.671 & 11.538 & 5.498 & 0.442 & 0.486 & 0.308 & 0.141 \\
\hline & 8 & 3.412 & 16.877 & 8.201 & 0.393 & 0.416 & 0.241 & 0.119 \\
\hline & 9 & 2.809 & 14.914 & 7.191 & 0.364 & 0.391 & 0.293 & 0.150 \\
\hline & 10 & 2.063 & 12.514 & 5.984 & 0.316 & 0.345 & 0.399 & 0.217 \\
\hline \multirow{8}{*}{2} & 11 & 1.278 & 9.424 & 4.220 & 0.246 & 0.303 & 0.643 & 0.373 \\
\hline & 12 & 0.887 & 5.709 & 2.568 & 0.282 & 0.345 & 0.927 & 0.504 \\
\hline & 13 & 2.020 & 11.810 & 5.164 & 0.304 & 0.391 & 0.407 & 0.208 \\
\hline & 14 & 1.523 & 11.154 & 5.203 & 0.256 & 0.293 & 0.540 & 0.319 \\
\hline & 15 & 2.219 & 12.760 & 5.804 & 0.319 & 0.382 & 0.371 & 0.191 \\
\hline & 16 & 1.754 & 11.226 & 5.242 & 0.293 & 0.335 & 0.469 & 0.259 \\
\hline & 17 & 3.381 & 12.262 & 5.732 & 0.518 & 0.590 & 0.243 & 0.101 \\
\hline & 18 & 0.993 & 7.240 & 3.592 & 0.272 & 0.276 & 0.828 & 0.503 \\
\hline \multirow{5}{*}{3} & 19 & 1.696 & 7.660 & 3.620 & 0.420 & 0.468 & 0.485 & 0.226 \\
\hline & 20 & 1.620 & 7.229 & 3.395 & 0.423 & 0.477 & 0.507 & 0.234 \\
\hline & 21 & 0.957 & 5.407 & 2.474 & 0.326 & 0.387 & 0.859 & 0.441 \\
\hline & 22 & 0.650 & 5.058 & 2.402 & 0.245 & 0.271 & 1.265 & 0.776 \\
\hline & 23 & 1.500 & 9.556 & 4.678 & 0.307 & 0.321 & 0.548 & 0.309 \\
\hline \multirow{10}{*}{4} & 24 & 2.041 & 11.535 & 5.542 & 0.341 & 0.368 & 0.403 & 0.212 \\
\hline & 25 & 1.535 & 10.570 & 5.201 & 0.286 & 0.295 & 0.536 & 0.315 \\
\hline & 26 & 1.020 & 8.352 & 4.019 & 0.235 & 0.254 & 0.806 & 0.511 \\
\hline & 27 & 1.336 & 10.788 & 5.256 & 0.241 & 0.254 & 0.615 & 0.390 \\
\hline & 28 & 1.123 & 9.629 & 4.757 & 0.231 & 0.236 & 0.732 & 0.481 \\
\hline & 29 & 1.348 & 8.174 & 3.931 & 0.318 & 0.343 & 0.610 & 0.332 \\
\hline & 30 & 1.407 & 8.566 & 3.889 & 0.301 & 0.362 & 0.584 & 0.310 \\
\hline & 31 & 1.113 & 19.051 & 4.685 & 0.077 & 0.238 & 0.738 & 0.484 \\
\hline & 32 & 1.332 & 7.760 & 3.677 & 0.326 & 0.362 & 0.617 & 0.327 \\
\hline & 33 & 1.491 & 16.755 & 4.032 & 0.117 & 0.370 & 0.551 & 0.290 \\
\hline
\end{tabular}

Table 4. Bed material size of Dizin River (mean of subreaches).

\begin{tabular}{cccccc}
\hline $\begin{array}{c}\text { Sampling } \\
\text { date }\end{array}$ & $\begin{array}{c}\text { Discharge } \\
\left(\mathbf{m}^{\mathbf{3}} / \mathbf{s}\right)\end{array}$ & $\begin{array}{c}\boldsymbol{D}_{\text {5osurface }} \\
\mathbf{( \mathbf { m m } )}\end{array}$ & $\begin{array}{c}\boldsymbol{D}_{\mathbf{8} 4 \text { surface }} \\
\mathbf{( \mathbf { m m } )}\end{array}$ & $\begin{array}{c}\boldsymbol{D}_{\text {50subsurface }} \\
\mathbf{( \mathbf { m m } )}\end{array}$ & $\begin{array}{c}\boldsymbol{D}_{\mathbf{8 4} \text { subsurface }} \\
\mathbf{( \mathbf { m m } )}\end{array}$ \\
\hline $28-\mathrm{Jul}-2009$ & 0.8221 & 51.2 & 254 & 12.24 & 33.8 \\
\hline
\end{tabular}




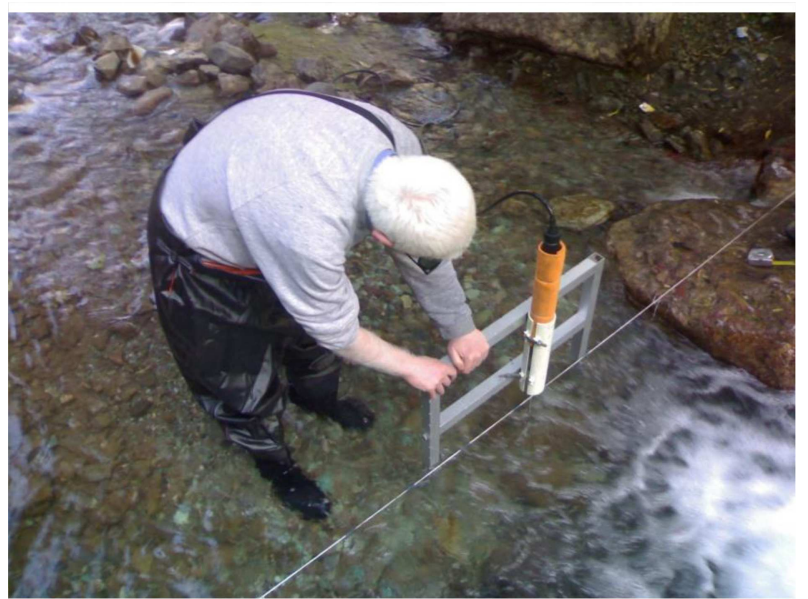

Figure 5. Velocity measurements.

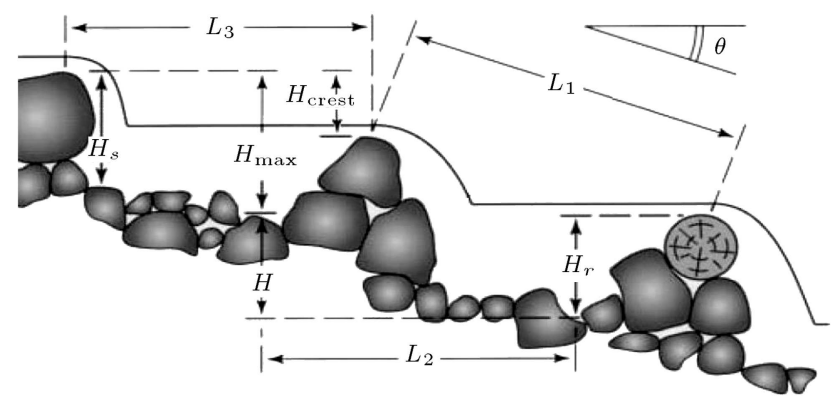

Figure 6. A schematic longitudinal section of a step-pool channel unit with micro-units included in the step.

the nonuniformity of bed material, hiding effect may appear strongly, which could cause erroneous results for $D_{50}$.

To evaluate hydraulic parameters, survey data were processed to evaluate step-pool geometry and water surface profile, based on the definitions, as shown in Figure 6. The principal quantities measured by several investigators are defined in Figure 6, where:

$L \quad$ Step-pool unit wavelength (crest-tocrest or pool-to-pool length),

$H \quad$ Total drop (of the bed) between pools,

$H_{\text {crest }}$ Total drop (of the bed) between steps,

$H_{\max } \quad$ Total drop (of the bed) between step and pool,

$H_{r} \quad$ Residual pool depth,

$H_{s} \quad$ Step height,

$L_{s} \quad$ step length, and
$L_{i} \quad$ Pool length.

Subscripts of $L$ identify studies that have used the indicated quantity: $1=$ Abrahams et al. (1995) and Zimmermann and Church (2001); 2 = Chin (1998,1999); 3 = Grant et al. (1990) and Chartrand and Whiting (2000) [2,6,23,26,39].

Reach slope was obtained by averaging the local slope values from the survey data along the thalweg. Table 5 describes the reach characteristics of Dizin River derived from survey data.

\section{Data analysis}

There are three common relationships to study uniform flow resistance: Chezy, Manning, and Darcy-Weisbach equations [15]. Owing to the complex nature of steppool morphology and roughness elements, which are large relative to water depth, channel geometry and flow characteristics are factors that affect flow resistance $[40,41]$. On the other hand, complex morphology of step-pool reaches makes velocity profile distribution in depth to be no longer valid [29]. Therefore, it is necessary to adopt an appropriate approach to including both channel geometry and flow characteristics, simultaneously. Dimensional analysis may offer a reasonable solution based on which the problem can be approached.

Based on the studies conducted on flow resistance in step-pool rivers $[27,28,42]$, it can be deduced that dimensionless Chezy coefficient $\left(C^{*}\right)$, which is basically the flow resistance coefficient, may be expressed as a function of the parameters in the following equation:

$$
f\left(C^{*}, S, H, L, D_{i}, R, q, g, \rho\right)=0
$$

where $S$ is the average slope of the river; $H$ is the step height; $L$ is the step length; $D_{i}$ is the diameter of the bed material size, $i$ is percentage of finer material; $R$ is hydraulic radius; $q$ is flow per channel width; $g$ is acceleration of gravity; and $\rho$ is fluid density.

Buckingham- $\pi$ theorem [43] is applied to evaluate dependency of the flow resistance in terms of dimensionless numbers. Herein, there are 9 variables and 3 dimensions. Thus, according to the Buckingham- $\pi$ theorem, there would be six dimensionless numbers as in Eq. (2):

$$
\pi_{1}=C^{*}, \quad \pi_{2}=S
$$

Table 5. Characteristics of Dizin River.

\begin{tabular}{cccccccccccccc}
\hline Longitude Latitude & Slope & $\begin{array}{c}\text { Reach length } \\
(\mathbf{m})\end{array}$ & $\begin{array}{c}\text { Number of } \\
\text { steps }\end{array}$ & $\begin{array}{c}\text { Width } \\
(\mathbf{m})\end{array}$ & $\begin{array}{c}\boldsymbol{H}_{\max } \\
(\mathbf{m})\end{array}$ & $\begin{array}{c}\boldsymbol{H}_{\text {crest }} \\
(\mathbf{m})\end{array}$ & $\begin{array}{c}\boldsymbol{H}_{\boldsymbol{r}} \\
(\mathbf{m})\end{array}$ & $\begin{array}{c}\boldsymbol{H}_{\boldsymbol{p}} \\
(\mathbf{m})\end{array}$ & $\begin{array}{c}\boldsymbol{H}_{\boldsymbol{s}} \\
(\mathbf{m})\end{array}$ & $\begin{array}{c}\boldsymbol{L}_{\mathbf{1}} \\
(\mathbf{m})\end{array}$ & $\begin{array}{c}\boldsymbol{L}_{\mathbf{2}} \\
(\mathbf{m})\end{array}$ & $\begin{array}{c}\boldsymbol{L}_{\mathbf{3}} \\
(\mathbf{m})\end{array}$ \\
\hline $51^{\circ}, 22^{\prime}$ & $36^{\circ}, 05^{\prime}$ & $11 \%$ & 120 & 12 & 10 & 0.61 & 0.50 & 0.24 & 0.35 & 0.36 & 4.56 & 4.65 & 4.59 \\
\hline
\end{tabular}




$$
\begin{aligned}
& \pi_{3}=D_{i}^{x_{1}} \cdot g^{y_{1}} \cdot \rho^{z_{1}} \cdot q \rightarrow\left\{\begin{array}{l}
x_{1}=-(3 / 2) \\
y_{1}=-(1 / 2) \Rightarrow \pi_{3}=\frac{q}{\sqrt{g D_{i}^{3}}} \\
z_{1}=0
\end{array}\right. \\
& \pi_{4}=D_{i}^{x_{2}} \cdot g^{y_{2}} \cdot \rho^{z_{2}} \cdot R \rightarrow\left\{\begin{array}{l}
x_{2}=-1 \\
y_{2}=0 \Rightarrow \pi_{4} \frac{R}{D_{i}} \\
z_{2}=0
\end{array}\right. \\
& \pi_{5}=D_{i}^{x_{3}} \cdot g^{y_{3}} \cdot \rho^{z_{3}} \cdot H \rightarrow\left\{\begin{array}{l}
x_{3}=-1 \\
y_{3}=0 \Rightarrow \pi_{5}=\frac{H}{D_{i}} \\
z_{3}=0
\end{array}\right. \\
& \pi_{6}=D_{i}^{x_{4}} \cdot g^{y_{4}} \cdot \rho^{z_{4}} \cdot L \rightarrow\left\{\begin{array}{l}
x_{4}=-1 \\
y_{4}=0 \Rightarrow \pi_{6}=\frac{L}{D_{i}} \\
z_{4}=0
\end{array}\right.
\end{aligned}
$$

The above dimensionless numbers may be reduced to Eq. (3), after simplifying:

$$
\begin{aligned}
C^{*} & =f\left(S, \frac{q}{\sqrt{g D_{i}^{3}}}, \frac{R}{D_{i}}, \frac{H}{L}\right) \Rightarrow \\
C^{*} & =f\left(S, q^{*}, \frac{R}{D_{i}}, \frac{H}{L}\right) .
\end{aligned}
$$

From Eq. (3), it is concluded that:

$$
q^{*}=\frac{q}{\sqrt{g D_{i}{ }^{3}}} .
$$

In this research, the flow resistance coefficient was extracted using the Darcy-Weisbach equation because of the following reasons:

For step-pool rivers, there is no general agreement, formula, or theorem on flow resistance to be valid for any given river [27]. Although flow in steep rivers is locally unsteady and non-uniform, it is possible to macroscopically assume the flow as uniform. By this assumption and considering the mean depth, slope, and hydraulic parameters, it is possible to calculate velocity in the given reach [44]:

1. In all previous studies conducted on flow resistance in step-pool rivers, uniform flow equations were applied to measure flow resistance (i.e., available data from other researchers are based on uniform flow equations);

2. Aplet (1983) emphasized that measuring the step drops using the Gauckler Manning's or DarcyWeisbach equations cannot provide satisfactory results; yet, these equations are applied to all flows; therefore, the present research is no exception [45].
The Darcy-Weisbach velocity equation was initially developed for flow in pipes. Nevertheless, it is widely applied to flat rivers with rigid contours as follows:

$$
\frac{V}{\sqrt{8 g R S_{f}}}=\sqrt{\frac{1}{f}}=C *
$$

where $V$ is the average flow velocity in $\mathrm{ms}^{-1}, g$ is the acceleration due to gravity in $\mathrm{ms}^{-2}, R$ is the hydraulic radius in $m, S_{f}$ is the energy slope, dimensionless, and $f$ is the observed Darcy-Weisbach roughness coefficient, dimensionless.

After separately analyzing $C^{*}$ variations with respect to each of $H / L, R / D_{84}, q^{*}$, and $S$ parameters, it can be claimed that variations of these parameters have the highest correlation with $C^{*}$ when applying exponential functions.

For a better analysis of exponential and logarithmic functions, the approach proposed by Maxwell and Papanicolaou (2001) was applied [46]. In this way, the equation of the resistance coefficient can be rewritten as general Eqs. (6) and (7):

$$
\begin{aligned}
& C^{*}=a \cdot \log \left((H / L) /\left(R / D_{i}\right)\right)+b, \\
& C^{*}=a \cdot(H / L)^{e} \cdot\left(R / D_{i}\right)^{f} .
\end{aligned}
$$

A comparison between the results shown in the table reveals that the exponential function involves a higher coefficient of correlation. Then, the variation function for $C^{*}$ is defined as in the generalized Eq. (8):

$$
C^{*}=a \cdot q^{* b} \cdot S^{d} \cdot\left(\frac{H}{L}\right)^{e} \cdot\left(\frac{R}{D_{i}}\right)^{f} .
$$

Values of cross-sectional area, top width, and hydraulic radius from Tables 1 to 3 were averaged for each subreach on the day of measurement (Table 6). In order to expand the database in the semi-arid environment, Ammameh River database was also added to the existing one [47].

A nonlinear regression analysis of the above data was conducted to evaluate the best fit curve as follows:

$$
\begin{aligned}
& C^{*}=0.463 \cdot q^{* 0.713} \cdot S^{-0.406} \cdot\left(\frac{H}{L}\right)^{0.23} \cdot\left(\frac{R}{D_{84}}\right)^{-0.963}, \\
& R^{2}=0.952 .
\end{aligned}
$$

Computed values of $C^{*}$ from Eq. (9) are plotted versus the measured values obtained from Eq. (5) in Figure 7.

To validate Eq. (9), field measurement data of Comiti et al. (2007) [28] for Rio Cordin were applied to estimate the measured and estimated values for $C^{*}$. Figure 7 indicates that the measured and estimated 
Table 6. The estimated value of hydraulic and geometric characteristics in the studied reach.

\begin{tabular}{cccccc}
\hline River & $\boldsymbol{C}^{*}$-meseared & $\boldsymbol{q}^{*}$ & $\boldsymbol{S}$ & $\boldsymbol{H} / \boldsymbol{L}$ & $\boldsymbol{R} / \boldsymbol{D}_{\mathbf{8 4}}$ \\
\hline \multirow{4}{*}{ Dizin } & 0.185 & 0.070 & 0.1405 & 0.1738 & 0.479 \\
& 0.2204 & 0.1050 & 0.1405 & 0.1738 & 0.5473 \\
& 0.2819 & 0.1692 & 0.0922 & 0.1784 & 0.7146 \\
& 0.3170 & 0.2398 & 0.0922 & 0.1784 & 0.8244 \\
& 0.3056 & 0.2825 & 0.0922 & 0.1784 & 0.9361 \\
& 0.6241 & 0.3332 & 0.0726 & 0.1203 & 0.5665 \\
& 0.4836 & 0.3838 & 0.0726 & 0.1203 & 0.9599 \\
& 0.4228 & 0.1533 & 0.1128 & 0.1868 & 0.5033 \\
& 0.5073 & 0.1997 & 0.1128 & 0.1868 & 0.4478 \\
& 0.3965 & 0.2361 & 0.1128 & 0.1868 & 0.6435 \\
& & & & & \\
& 0.1502 & 0.0225 & 0.105 & 0.138 & 0.282 \\
& 0.3127 & 0.1179 & 0.105 & 0.138 & 0.525 \\
& 0.2944 & 0.2077 & 0.105 & 0.138 & 0.774 \\
\hline \multirow{5}{*}{ Amameh } & & & & &
\end{tabular}

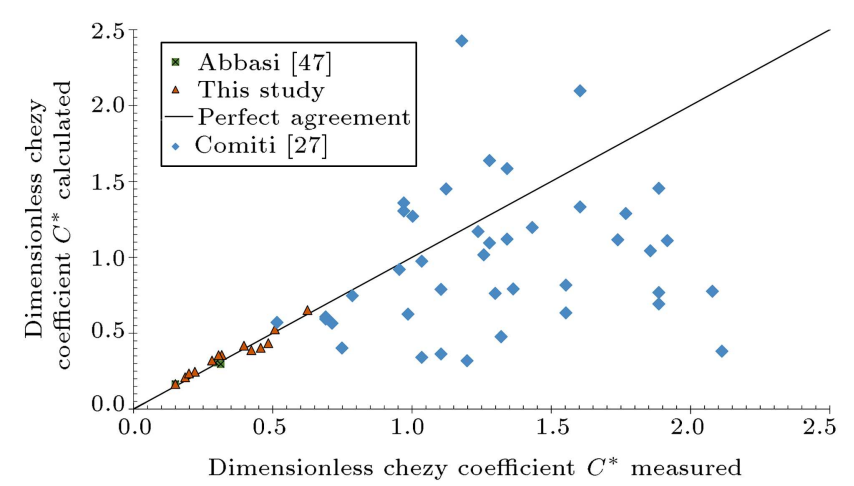

Figure 7. A comparison of measured $C^{*}$ values estimated using the Darcy-Weisbach equation and those calculated using Eq. (9).

$C^{*}$ values have a reasonable scatter about the best fit line due to the diverse environments of the dataset. However, based on possible speculations, although Eq. (9) was derived from the semi-arid dataset, it could also give reasonable results for humid dataset.

In the study of Comiti et al. (2007) [28], the variations of $C^{*}$ in the study are different. This may be reflected by different parameters among which $R / D_{84}$ and $q^{*}$ are the most important ones. $q^{*}$ depends on $q / \sqrt{ }\left(R D_{84}\right)$, in which $q$ plays a key role. This is because the results of the studied area point to the range of $0.4 \sim 0.822 \mathrm{~m}^{3} / \mathrm{s}$, while, in Comiti's study, it is in a range of $0.08 \sim 1.86 \mathrm{~m}^{3} / \mathrm{s}$. This is also confirmed by the ratio of $R / D_{84}$ since this ratio varies in $0.448 \sim 0.96$ in this study, while, in Comiti's study, the ratio varies in $0.1 \sim 1.176$. Furthermore, $D_{84}$ for the studied reach varies in $0.3 \sim 0.65$ meter, which is roughly the same as Comiti's. This, in turns, shows that high $q$ values are reflected by high $R$ values, increasing the range of variations in Comiti's database.

Sensitivity analysis was also conducted to have an in-depth understanding of the effect of each term in Eq. (9) for $C^{*}$ estimations (Table 7 ). The analysis was preceded by eliminating each term from Eq. (9) except for the term, including $q^{*}$. This is due to the fact that, in the absence of $q^{*}$, there will be no discharge in the river. Errors have also been estimated by Eq. (10):

$$
\mathrm{RSME}=\sqrt{\frac{\sum_{i=1}^{n}\left(C *_{\text {Measured }}-C *_{\text {estimated }}\right)^{2}}{n}},
$$

where $C_{\text {Measured }}^{*}$ is measured values, $C_{\text {estimated }}^{*}$ is estimated values from Eq. (9), and $n$ is number data. Table 7 shows that estimated $C^{*}$ may have the highest sensitivity with respect to elimination of $\frac{R}{D_{84}}$.

\section{Conclusion}

This study presented a formula to estimate the resistance coefficient in the mountain stream as a function of $H / L, R / D_{84}, q^{*}$, and $S$ for the measured data of Dizin river located in a semi-arid environment. Results 
Table 7. Sensitivity analysis of the effect of each term in Eq. (9) for $\mathrm{C}^{*}$ estimation.

\begin{tabular}{ccccccc}
\hline $\boldsymbol{a}$ & $\boldsymbol{b}$ & $\boldsymbol{d}$ & $\boldsymbol{e}$ & $\boldsymbol{f}$ & $\boldsymbol{R}^{\mathbf{2}}$ & RMSE \\
\hline 0.536 & 0.797 & $\ldots$ & -0.259 & -.0957 & 0.941 & 0.032 \\
0.311 & 0.715 & -0.383 & $\ldots$ & -0.961 & 0.950 & 0.029 \\
0.216 & 0.388 & -0.327 & -0.205 & $\ldots$ & 0.636 & 0.079 \\
\hline
\end{tabular}

showed reasonable agreement between observed and estimated values of $C^{*}$. Data obtained from our observations of a river located in a humid environment were also used to investigate the validation of the relationship. It is suggested that estimated and measured values of $C^{*}$ for these data seem to be in reasonable agreement. Accordingly, it can be judged that flow resistance parameters of the mountain stream in semiarid and humid environments may be comparable.

As $H / L$ and $q^{*}$ increase, $C^{*}$ increases and $f$ decreases. Conversely, an increase in $S$ and $R / D_{84}$ may result in a decrease in $C^{*}$ and, hence, an increase in the values of $f$. Furthermore, based on the exponents obtained in Eq. (9), $R / D_{84}, q^{*}, S$, and $H / L$ seem to be very significant.

\section{Nomenclature}

$\begin{array}{ll}H_{\text {crest }} & \text { Total drop (of the bed) between steps } \\ H_{\text {max }} & \text { Total drop (of the bed) between step } \\ & \text { and pool } \\ H_{r} & \text { Residual pool depth } \\ H_{s} & \text { Step height } \\ L_{s} & \text { Step length } \\ L_{i} & \text { Pool length } \\ C^{*} & \text { Dimensionless Chezy coefficient } \\ n & \text { Number data } \\ V & \text { Average flow velocity } \\ g & \text { Acceleration due to gravity } \\ R & \text { Hydraulic radius } \\ S_{f} & \text { Energy slope } \\ f & \text { Darcy-Weisbach roughness coefficient } \\ S & \text { Average slope of the river } \\ L & \text { Step length } \\ D i & \text { Diameter of the bed material size } \\ q & \text { Percentage of material finer } \\ q & \text { Flow per channel width } \\ \rho & \text { Fluid density }\end{array}$

\section{References}

1. Montgomery, D.R. and Buffington, J.M. "Channelreach morphology in mountain drainage basins", Geological Society of America Bulletin, 109(5), pp. 596611 (1997).
2. Grant, G., Swanson, F., and Wolman, G. "Pattern and origin of stepped-bed morphology in high-gradient streams, Western Cascades, Oregon", Geological Society of America Bulletin, 102, pp. 340-352 (1990).

3. Chin, A. "The morphologic structure of step-pools in mountain streams", Geomorphology, 27, pp. 191-204 (1999).

4. Grant, G.E. "Hydraulics and sediment transport dynamics controlling step-pool formation in high gradient streams: a flume experiment", In Dynamics and Geomorphology of Mountain Rivers, Ergenzinger, P., Schmidt, K.H. (eds), Lecture Notes in Earth Sciences, 52, Springer-Verlag, Berlin, pp. 241-250 (1994).

5. Chin, A. "Step-pools in stream channels", Progress in Physical Geography, 13(3), pp. 391-407 (1989).

6. Chartrand, S. and Whiting, P. "Alluvial architecture in headwater streams with special emphasis on step-pool topography", Earth Surface Processes and Landforms, 25, pp. 583-600 (2000).

7. Maxwell, A.R., Papanicolaou, A.N., Hotchkiss, R.H., Barber, M.E., and Schafer, J. "Step pool morphology in high gradient countersunk culverts", Transportation Research Record, 1743, pp. 49-56 (2001).

8. Yu, G.A., Wang, Z.Y., Zhang, K., Xuehua, D., and Chang, T.C. "Restoration of an incised mountain stream using artificial step-pool system", Journal of Hydraulic Research, 48(2), pp. 178-187 (2010). DOI: $10.1080 / 00221681003704186$

9. Chin, A., et al. "Linking theory and practice for restoration of step-pool streams", Environmental Management, 43, pp. 645-661 (2009). DOI:10.1007/s00267008-9171

10. Nikseresht, A.H., Talebbeydokhti, N., and Rezaei, M.J. "Numerical simulation of two-phase flow on steppool spillways", Scientia Iranica, 20(2), pp. 222-230 (2013).

11. Chin, A. and Wohl, E. "Toward a theory for step pools in stream channels", Progress in Physical Geography, 29(3), pp. 275-296 (2005).

12. Whittaker, J.G., Sediment Transport in Step-pool Streams, Sediment Transport in Gravel Bed Rivers, pp. 545-579 (1987).

13. David, G.C.L., Wohl, E.E., Yochum, S.E., and Bledsoe, B.P. "Controls on spatial variations in flow resistance along steep mountain streams", Water Resources Research, 46(3), pp. 1-21 (2010). DOI: 10.1029/2009WR008134

14. Canovaro, F. and Solari, L. "Flow resistance associated to step-pool pattern", GBR6th Gravel Bed River Conference (GBR6): Poster Abstract, pp. 45-47 (2007). 
15. Rickenmann, D. and Recking, A. "Evaluation of flow resistance in gravel-bed rivers through a large field dataset", Water Resources Research, 47, pp. 1-23 (2011). DOI: 10.1029/ 2010WR009793

16. Zimmermann, A. "Flow resistance in steep streams: an experimental study", Water Resources Research, 46(9), pp. 1-18 (2010). DOI:10.1029/2009WR007913

17. Judd, H.E., A Study of Bed Characteristics in Relation to Flow in Rough, High- Gradient, Natural Channels, UTAH State University, Logan, Utah (1963).

18. Chartrand, S.M., Jellinek, M., Whiting, P.J., and Stamm, J. "Geometric scaling of step-pools in mountain streams: observations and implications", Geomorphology, 129, pp. 141-151 (2011).

19. Curran, J.H., and Wohl, E. "Largewoody debris and flow resistance in step-pool channels, Cascade range, Washington", Geomorphology, 51, pp. 141-157 (2003).

20. McFarlane, W.A., and Wohl, E.E. "Influence of step composition on step geometry and flow resistance in step-pool streams of the Washington Cascades", Water Resources Research, 39(2), pp. 1-13 (2003).

21. Wilcox, A. and Wohl, E.E. "Flow resistance dynamics in step-pool channels: 1. Large woody debris and controls on total resistance", Water Resources Research, 42(5), pp. 1-16 (2006).

22. Wilcox, A., Nelson, J.M., and Wohl, E.E. "Flow resistance dynamic in step-pool channels: 2. Partitioning between grain, spill, and woody debris flow resistance", Water Resources Research, 42(2005WR004278), pp. 114 (2006).

23. Zimmermann, A. and Church, M. "Channel morphology, gradient profiles and bed stresses during flood in a step-pool channel", Geomorphology, 40, pp. 311-327 (2001).

24. Church, M. and Zimmerman, A. "Form and stability of step-pool channels: research progress", Water Resources Research, 43(W03415), pp. 1-21 (2007).

25. Wilcox, A.C., Wohl, E.E., Comiti, F., and Mao, L. "Hydraulics, morphology, and energy dissipation in an alpine step-pool channel", Water Resources Res., 47(W07514) (2011).

26. Abrahams, A.D., Li, G., and Atkinson, J.F. "Step-pool streams: adjustment to maximum flow resistance", Water Resources Research, 31(10), pp. 2593-2602 (1995).

27. Lee, A.J. and Ferguson, R. "Velocity and flow resistance in step-pool streams", Geomorphology, 46, pp. 59-71 (2002).

28. Comiti, F., Mao, L., Wilcox, A., Wohl, E., and Lenzi, M. "Field-derived relationships for flow velocity and resistance in high-gradient streams", Journal of Hydrology, 21(3), pp. 48-62 (2007).

29. Ferguson, R. "Flow resistance equations for gravel and boulder bed streams", Water Resources Research, 43(W05427), pp. 1-12 (2007).
30. Turowski, J.M., Yager, E.M., Badoux, A., Rickenmann, D., and Molnar, P. "The impact of exceptional events on erosion, bedload transport and channel stability in a step-pool channel", Earth Surface Processes and Landforms, 34, pp. 1661-1673 (2009). DOI: 10.1002 /esp. 1855

31. D'Agostino, V. and Michelini, T. "On kinematics and flow velocity prediction in step-pool channels", Water Resour. Res., 51, pp. 4650-4667 (2015). DOI: 10.1002/2014WR016631.

32. Sindelar, C. and Smart, G. "Transition flow in steppool systems: Pressure distributions and drag", Journal of Hydraulic Engineering, 142(10), pp. 1779-1988 (2016). DOI: 10.1061/(ASCE)HY.1943-7900.0001165

33. Maddahi, M.R., Afzalimehr, H., and Rowinski, P.M. "Flow characteristics over a gravel bedform: Kaj River case study", Acta Geophysica, 64(5), pp. 1779-1796 (2016).

34. SonTek, Flow Tracker Handheld ADV Technical Documentation, San Diego, Calif (2001).

35. Rehmel, M. "Application of acoustic Doppler velocimeters for stream flow measurements", Journal of Hydraulic Engineering, 133(12), pp. 1433-1438 (2007). DOI:10.1061/(ASCE)0733-9429(2007)133:12(1433)

36. Wolman, M.G. "A method of sampling coarse riverbed material", Trans. Am. Geophys. Union, 35(6), pp. 951-956 (1954).

37. Milhous, R.T., Hogan, S.A., Abt, S.R., and Watson, C.C. "Sampling river-bed material: the barrel sampler", Rivers, 5, pp. 239-249 (1995).

38. Shvidchenko, A.B. and Pender, G. "Flume study of the effect of relative depth on the incipient motion of coarse uniform sediments", Water Resources Research, 36(2), pp. 619-628 (2000).

39. Chin, A. "On the stability of step-pool mountain streams", Journal of Geology, 106, pp. 59-69 (1998).

40. Rickenmann, D. "Hyper concentrated flow and sediment transport at steep slopes", Journal of Hydraulic Engineering ASCE, 117(11), pp. 1419-1439 (1991).

41. Aberle, J. and Smart, G.M. "The influence of roughness structure on flow resistance on steep slopes", Journal of Hydraulic Research, 41(3), pp. 259-269 (2003).

42. Bathurst, J.C. "At-a-site variation and minimum flow resistance for mountain rivers", Journal of Hydrology, 269, pp. 11-26 (2002).

43. Buckingham, E. "On physically similar systems: illustrations of the use of dimensional equations", Phys. Rev., 4(4), pp. 345-376 (1914).

44. Judd, H.E. and Peterson, D.F. "Hydraulics of large bed element channels", Report PRWG 17-6, Utah Water Research Laboratory, Utah State University (1969).

45. Apelt, C.J. "Hydraulics of minimum energy culverts and bridge waterways", Australian Civil Engineering Transactions, I.E. Aust., CE25 2, pp. 89-95 (1981). 
46. Maxwell, A.R. and Papanicolaou, A. "Step-pool morphology in high-gradient streams", International Journal of Sediment Research, 16(3), pp. 380-390 (2001).

47. Abbasi, F. and Tabatabai, M.R.M. "Field investigation of hydraulic and morphologic relationships along steppool reach", Iran-Watershed Management Science \& Engineering, 3(7), pp. 35-42 (2009).

\section{Biographies}

Ahmad Tahershamsi was born in 1951 in Qom, Iran. He graduated in Civil Engineering in 1976 from Amirkabir University of Technology, Iran. In 1988, he received his $\mathrm{PhD}$ degree from the Department of Civil and Structural Engineering at the Institute of Science and Technology, University of Manchester (UMIST), UK. From 2000-2001, he also attended San Diego State University, California, USA, as an Adjunct Professor. Currently, Dr. Tahershamsi is an Associate Professor of Hydraulic Engineering at Amirkabir University of Technology, Tehran, Iran. His scientific interests include hydraulic structures, dam breaks, environmental hydraulics, and ground water engineering. Dr. Tahershamsi is the author of 40 papers published in national and international journals and 100 papers presented at national and international conferences. He is also the author of a book entitled "Computational Hydraulics" in Persian.

Mohammad Reza Majdzadeh Tabatabai was born in 1963 in Tehran, Iran. He graduated in Civil Engineering from King's College, University of London in 1989 and did his MSc in Water Resources Engineering in Queens Mary and Westfield College of University of London in 1991. He also did his PhD in Modelling of Fluvial Processes in University of East
Anglia in Britain and attended as a Visiting Professor at Civil Engineering Department, University of British Columbia (2003-2004). He is currently an Assistant Professor of River Engineering in Civil, Water and Environmental Engineering Department of Shahid Beheshti University. Dr Tabatabai's research interests are focused on the processes controlling channel morphology, secondary flows and shear stress distributions, flow resistance, bedload transport, bank erosion due to surface and subsurface flows, river bed armoring processes, meander mechanisms, flow and sediment transport processes in meandering channels with overbank flow, and the development of regime equations for mobile gravel-bed rivers. The recent applied works involve the design of flood alleviation schemes and the restoration of natural channels for the National Rivers Authority in Iran, determination of channel forming discharges for Iranian National Rivers Authority, applications of POT for estimations of flood return period and the relationship between return periods estimated from POT and AMS, Optimized flood management approach, flood risk assessment in urban floodplain, GIS application in flood zone mapping, reservoir sedimentation and flushing numerical simulation, numerical simulation of settling basin, hydraulic design of river intake, river and reservoir management and integrated water resources management.

Abbas Torabizadeh was born in Ahwaz, Iran in 1983. He received his BS degree in Civil Engineering in 2007 from the Shahid Chamran University of Ahwaz and his MS degree in River Engineering from Shahid Chamran University, Ahwaz, Iran in 2009. His main research interests lie in the energy dissipation in mountain river. He also has interests in flow resistance in step-pool streams. 